\title{
UNA EXPERIENCIA DE TRABAJO COLABORATIVO CON OFFICE LIVE WORKSPACE
}

\author{
María Pilar Flores-Asenjo: Universidad Católica San Antonio de Murcia. España. \\ pflores@pdi.ucam.edu.
}

\section{RESUMEN}

Al situar al estudiante en el centro del proceso de enseñanza-aprendizaje, se produce la necesidad de realizar un cambio metodológico que potencie el papel activo del estudiante. Las nuevas Tecnologías ofrecen nuevas posibilidades en este contexto. En esta experiencia se intenta involucrar a un pequeño grupo de estudiantes en este proceso, mediante el análisis, evaluación, elección y uso de "web colaborativa" para el desarrollo de un trabajo de investigación en equipo para, posteriormente, evaluar el grado de adquisición de competencias transversales. El trabajo se desarrolla con un pequeño grupo de alumnos de Administración de Empresas poco habituados al uso de las TIC. Partiendo de un debate a cerca de las dificultades en la gestión y planificación de un trabajo en equipo, se les encomienda que investiguen sobre la conceptualización y características de la Web 2.0. Su puesta en común mediante debate, lleva a evaluar los distintos ejemplos de casos reales encontrados, su uso en el nuevo Espacio Europeo de Educación Superior y las distintas posibilidades gratuitas disponibles, así como la elección de una de ellas según los requerimientos del grupo. Una vez desarrollado el trabajo de investigación propuesto, se realiza un nuevo debate en el que se analizan las competencias adquiridas. En este caso el alumno participa de todo el proceso junto al docente y se observa que se produce una mejor percepción de los resultados de aprendizaje y se facilita la evaluación de las competencias transversales.

PALABRAS ClAVE: TIC - Aprendizaje activo - Web 2.0 - Cloud Computing Competencia Transversal

\footnotetext{
${ }^{1}$ Autor Correspondiente

$\mathbf{M}^{a}$ Pilar Flores-Asenjo: Profa. Dra. Titular. Universidad Católica San Antonio de Murcia. Guadalupe, España
} 


\title{
AN EXPERIENCE OF COLLABORATIVE WORK WITH OFFICE LIVE WORKSPACE
}

\begin{abstract}
Placing the student in the centre of the teaching-learning process makes a methodological change that strengthens the active role of the student. New technologies offer new possibilities in this context. This experience seeks to involve a small group of students in this process, through analysis, evaluation, selection and use of "collaborative web" for the development of a research work in a team for, later, to assess the degree of the acquisition of cross-curricular skills. The work is carried out with a small group of students from Business Administration little accustomed to the use of new technologies. Starting from a debate about the difficulties in the management and planning of a team work, they have been assigned to investigate on the conceptualization and characteristics of Web 2.0: evaluate different examples of actual cases found, their use in the new European Higher Education Area and the various possibilities available, as well as the choice of one of them according to the requirements of the group. Once developed the research work proposed, we take a new debate in which we analyze the skills acquired. The student participates in the whole process together with the teacher. It produces a better perception of the learning outcomes and facilitates the assessment of cross-curricular skills.
\end{abstract}

KEY WORDS: ICT - Active learning- web 2.0 - cloud computing - Cross-curricular skill

\section{INIRODUCCIÓN}

\subsection{El trabajo en equipo y colaborativo y las nuevas tecnologías en el espacio europeo de educación superior}

Con motivo de la Declaración de Bolonia de 1999 se configura un nuevo Espacio Europeo de Educación Superior (EEES) que implica un cambio en el papel del profesor para adaptarse a nuevos métodos docentes y dotar al alumno de ciertas aptitudes que trascienden el conocimiento académico. El estudiante aparece ahora en el centro del proceso de enseñanza-aprendizaje, se focalizan los estudios en las competencias que debe poseer el recién egresado, potenciando el saber hacer del estudiante, la iniciativa y el aprendizaje autónomo, según marcan los descriptores de Dublín (Joint Quality Initiative, 2004). 
El EEES prioriza la competencia transversal "trabajo colaborativo", pues el principal objetivo es la formación del alumnado en la capacitación de construir sus propios conocimientos, para superar así el sistema pedagógico tradicional. Como señala Carrasco Embuena (Carrasco Embuena, 2007), las nuevas propuestas acentúan el concepto de aprendizaje como proceso continuo y consideran la autonomía de los estudiantes como una contingencia necesaria orientada a desarrollar sus habilidades. Las diferentes técnicas que se utilizan para el aprendizaje colaborativo permiten que los alumnos compartan información y conocimiento mejorando sus habilidades de comunicación y facilitando la confianza en sí mismos sobre su capacidad para definir problemas y aplicar sus conocimientos para resolverlos (Yazici, 2004).

El escenario actual en el que se produce una permanente renovación tecnológica remodela diferentes campos disciplinarios y los escenarios de la sociedad. En este contexto, la formación docente debe incluir una parcela de formación sobre las TIC, orientada a la realidad práctica, a la creación de materiales interactivos, su utilización para su propio aprendizaje, para su realización con los alumnos, con otros profesores, etc. McClintock (McClintock, 2000) concluye que "las nuevas tecnologías, específicamente los sistemas telemáticos, son medios interesantes para introducir pedagogías alternativas y potenciar cambios en las estructuras educativas".

Internet ha sufrido una revolución con la aparición de la web 2.0 o web social. Este conjunto de tecnologías para la creación social de conocimiento, incorpora tres características esenciales: tecnología, conocimiento y usuarios; y se caracteriza por la creación colectiva de contenidos, el establecimiento de recursos compartidos y el control de la calidad de forma colaborativa entre los usuarios (Ribes, 2007). Por tanto, generan espacios de comunicación idóneos para el desarrollo de habilidades tales como el pensamiento crítico, la autonomía, la iniciativa, el trabajo colaborativo y/o la responsabilidad individual (Bruns \& Humphreys, 2005) y son un nuevo marco práctico para la socialización y la culturización de los jóvenes (Pérez Tornero, 2008).

La comunicación a través de internet posibilita la interacción permanente en los proyectos de investigación (globalización de la investigación), además de establecer redes educativas para el intercambio de experiencias de innovación docente, permite superar barreras espaciales y temporales, lo que facilita tanto la búsqueda y el procesamiento de información como el desarrollo original de contenidos por parte de los alumnos. Además, nos ofrece como docentes una amplia gama de posibilidades para innovar en los patrones tradicionales de aprendizaje (Area, 2005); (Blok et al., 2002); (Ezziane, 2007).

Las actividades que combinan las nuevas metodologías docentes con las TICs facilitan el aprendizaje y permiten el desarrollo de la capacidad para la resolución de problemas y para la toma de decisiones (López \& Villafañe, 2010), la capacidad para trabaiar en eauipo v la cabacidad de ablicar los conocimientos en la práctica. Además. 
Uno de los objetivos para los docentes que más explícitamente se subraya en el nuevo marco formativo es el de no sólo enseñar, sino también enseñar a aprender, lo que pone de relieve la necesidad de adoptar diferentes metodologías docentes que generen mayor motivación (Arquero \& Jiménez, 1999). Para implementar un ambiente de aprendizaje efectivo Jonassen (Jonassen, 1994) propone una serie de características generales de aprendizaje como enfatizar la construcción del conocimiento en vez de la reproducción del mismo, fomentar la reflexión basada en la experiencia, y apoyar la construcción colaborativa del conocimiento a través de la negociación social y no la competencia.

Tanto la utilización de las TIC como el "trabajo colaborativo" permite a los alumnos el desarrollo de oportunidades de aprendizaje que se apartan completamente del modelo pedagógico basado en memorizar y consigue que el alumno aprenda inicialmente desde la práctica y desde sus propias hipótesis mediante la participación. Además, "aprender haciendo" tiene el beneficio adicional de favorecer la memoria a largo plazo (Sousa, 2001).

\section{METODOLOGÍA}

\subsection{Desarrollo y evaluación de la experiencia}

Teniendo en cuenta lo analizado anteriormente a cerca del uso de las TIC y del trabajo en equipo y colaborativo, se llevó a cabo un experimento de realización en grupo de un trabajo de investigación a través de web 2.0. El equipo estaba formado por alumnos poco habituados al uso de las nuevas tecnologías, debido a su edad (media superior a 45 años), y con problemas para coincidir espacial y temporalmente para llevar a cabo el trabajo de investigación.

El objetivo principal fue poner en práctica estrategias de aprendizaje, de trabajo y de estudio en un entorno virtual, a partir de la adquisición de habilidades expresadas en términos de competencias genéricas en TIC, en un escenario de trabajo cooperativo, siendo los objetivos competenciales: el fomento de la reflexión crítica sobre el impacto de las tecnologías de la información y la comunicación, la adquisición de habilidades de trabajo en equipo en entornos virtuales, la búsqueda y selección de la información en red, el análisis, tratamiento e interpretación de información y el desarrollo y gestión de un trabajo en equipo en red.

Para favorecer el éxito del proyecto colaborativo es necesario realizar una adecuada planificación donde se definan los objetivos a alcanzar, los contenidos a tratar y la metodología a aplicar para su desarrollo (Echazarreta et al., 2009). El docente está presente en cada uno de los pasos del alumno como orientador y guía de la experiencia de aprendizaje, lo que supone haber realizado previamente una correcta nrogramación de la misma. 


\section{ANÁLISIS Y DISCUSIÓN}

En la primera sesión, en la que se plantea el problema, se establece también la secuencia de trabajo presencial y los objetivos a alcanzar por los alumnos. Se debaten los posibles problemas de planificación y organización del trabajo y cómo los entornos virtuales pueden servir de ayuda para resolverlos. En esta sesión se emplaza a los alumnos a que investiguen las posibilidades de las TIC, que busquen en la red casos reales de trabajo y colaboración en equipo exitosos, así como información sobre la EEES y el trabajo por competencias.

En una segunda sesión, se expone la información recopilada por los alumnos, entre la que destaca el caso del escándalo de los diputados británicos en la que se exponen al público cerca de 500.000 documentos y, mediante una hoja de Google Docs, los lectores leen y localizan irregularidades que luego son publicadas por un periódico. Se interpretan y analizan otros casos y herramientas utilizadas, para llegar a la conclusión de la posibilidad de utilizar una web colaborativa para el desarrollo del trabajo de investigación. Esto supone que los alumnos investiguen las ventajas y los inconvenientes de las diferentes alternativas gratuitas ( $\mathrm{y}$, por tanto, accesibles para todos los miembros) localizadas en Internet. Tras un debate, se elige una de ellas y se emplaza a los alumnos a una sesión para aprender a utilizarla.

La herramienta seleccionada fue Office Live Workspace: El servicio de Microsoft está integrado con un software gratuito para compartir escritorio llamado SharedView, con el cual pueden trabajar varias personas al mismo tiempo en un documento y mantener discusiones vía chat para coordinarse. Proporciona alertas de actividad personalizables "para mantenernos al tanto de lo que ocurre en nuestro espacio de trabajo" y "no sólo podemos compartir documentos, sino que Workspaces completos" (carpetas) con lo que el trabajo en grupo se hace "mucho más fácil y fluido".

Una vez que los alumnos tienen los rudimentos básicos para utilizar la herramienta seleccionada, comienza el trabajo de elaboración del proyecto de investigación. En esta fase los alumnos interiorizan actitudes básicas necesarias para un trabajo cooperativo virtual, como el compromiso, la transparencia, la constancia y el respeto. Practican habilidades relacionadas con la comunicación virtual y asíncrona, aprenden a organizarse, a revisar y ajustar la planificación, buscar y gestionar información, resolver conflictos, argumentar, buscar el consenso, tomar de decisiones, etc. Los alumnos aprenden de su propia experiencia, reforzando el aspecto motivador de esta metodología y fomenta la discusión, la defensa de argumentos y el acercamiento entre posturas; pero siempre que estén adecuadamente orientados y estén claros los objetivos a alcanzar con su trabajo.

La motivación, la capacidad de abordar problemas complejos, la participación activa y la implicación por medio de la distribución de roles son alqunos de los rasqos que 
Los estudiantes toman conciencia de la importancia de una buena planificación, de la diversidad de formas en las que se puede llevar a cabo y de las consecuencias que pueden tener en el óptimo desarrollo del trabajo en equipo. La colaboración fomenta que los estudiantes se sientan parte de una comunidad de aprendizaje, lo que favorece la construcción de conocimiento compartido y crea un espíritu comunitario (Garrison \& Anderson, 2005); (Tu, 2004).

En la evaluación de la experiencia a través de debate y puesta en común de resultados los alumnos confirmaron que fue una "experiencia motivadora" que les ayudó a "comprender la necesidad de la planificación del trabajo, el respeto y el compromiso en el trabajo en equipo y la utilidad de las nuevas tecnologías en su desarrollo". Además, los alumnos comprendieron y valoraron la importancia de la guía y orientación del docente, que "facilitó la comprensión de los objetivos a alcanzar para poder realizar una exitosa planificación del trabajo".

\section{CONCLUSIONES}

Las competencias transversales nacen de la intersección entre múltiples disciplinas y son fundamentales en el nuevo currículo universitario. El EEES prioriza la competencia transversal "trabajo colaborativo" con el objetivo de promover un aprendizaje autónomo.

El trabajo colaborativo facilita la formación de grupos heterogéneos, fomenta la responsabilidad de cada miembro y crea una interdependencia positiva que exige a los participantes habilidades comunicativas, relaciones simétricas y recíprocas y capacidad de resolución de las tareas. El alumno aprende la importancia de la planificación del trabajo y valora la programación de los objetivos que exige la constante orientación y guía del docente.

Las webs colaborativas brindan a sus usuarios la posibilidad de consultar, editar, corregir y reorganizar los contenidos existentes, creando una serie de sinergias que propician la capacidad de organización y planificación, la capacidad para la resolución de problemas y para tomar decisiones, la capacidad para trabajar en equipo, la habilidad en las relaciones personales y la capacidad crítica. Fomentan la participación en proyectos comunes al permitir reunir virtualmente a colaboradores que trabajan a distancia, y son una herramienta motivadora para el aprendizaje.

En la evaluación de las competencias adquiridas se concluyó, además, la alta satisfacción con los resultados conseguidos y la "posibilidad de aplicar esta experiencia en otras asignaturas". 


\section{REFERENCIAS}

Area, M. (2005). Las tecnologías de la información y comunicación en el sistema escolar. Una revisión de las líneas de investigación.

Arquero, J. L. \& Jiménez, S. M. (1999). Influencia del estudio de casos en la mejora del aprendizaje, adquisición de capacidades no técnicas y motivación en análisis contable. Revista de Enseñanza Universitaria Extraordinario, 1: 225-242.

Blok, H., R.; Oostdam, M.; Otter, E. \& Overmaat, M. (2002). Computer- assisted instruction in support of beginning reading instruction: A review. Review of Educational Research, 72: 101-130.

Carrasco Embuena, V. (2007). Diseño de modelos de coordinación docente-discente para los nuevos títulos de grado y postgrado en el marco EEES. Algunas aportaciones y propuestas En La multidimensionalidad de la educación universitaria. Redes de investigación docente- Espacio Europeo de Educación Superior. (Vol. I). Alcoy: Ed. Marfil.

Echazarreta, C; Prados, F.; Poch, J. \& Soler, J. (2009). La competencia El trabajo colaborativo: una oportunidad para incorporar las TIC en la didáctica universitaria. Descripción de la experiencia con la plataforma ACME (UdG). Revista sobre la sociedad del conocimiento, 8. Recuperado el 7 de junio de 2011, de www.uoc.edu/uocpapers/.../echazarreta_prados_poch_soler.html.

Ezziane, Z. (2007). Information technology literacy: Implications on teaching and learning. Educational Technology and Society, 10: 175-191.

Garrison, D.R. \& ANDERSON, T. (2005). El e-learning en el siglo XXI. Barcelona: Octaedro.

Gértrudix Barrio, M. (2006). Convergencia multimedia y Educación. ICONO, 14 (7). Recuperado el 6 de junio de 2011, de www.icono14.net/revista/num7/articulos/manuel_gertrudix.pdf.

Joint Quality Initiative. (2004). Shared 'Dublin' descriptors for the Bachelor's, Master's and Doctoral awards. Recuperado el 26 de mayo de 2011, de www.jointquality.nl/content/descriptors/CompletesetDublinDescriptors.doc.

Jonassen, D.H. (1994). What might constructivism mean in education? Penn State Education Summit: Issues on the Forefront. University Park, PA.

López Jiménez, I. E. \& Villafañe Rodríguez, C. (2010-11). La Integración de 
Mcclintock, R. (2000). Prácticas pedagógicas emergentes. El papel de las tecnologías de la información y la comunicación. Cuadernos de Pedagogía, 290: 74-77.

Pérez Tornero, J. M. (2008). Teacher Training Curricula for Media and information Literacy". International Expert Group Meeting. Paris: UNESCO Headquarters,.

Recuperado el 25 de mayo de 2011 http://portal.unesco.org/ci/en/files/27068/12133527103Background_Paper.d oc/Background \%2BPaper.doc.

Relieve. (2011). Revista Electrónica de Investigación y Evaluación Educativa, 11. Recuperado el 7 de junio de 2011, de www.uv.es/RELIEVE/v11n1/RELIEVEv11n1_1.htm

Ribes, X. (2007). La Web 2.0, el valor de los metadatos y de la inteligencia colectiva. Telos, 73.

Salinas, J. (1997). Nuevos ambientes de aprendizaje para una sociedad de la información. Revista Pensamiento Educativo, 20: 81-104.

Sousa, D. A. (2001). How the brain learns. A classroom teacher's guide. (2nd ed.). California: Corwin Press Thousand Oaks.

$\mathrm{Tu}$, C. (2004). Online collaborative learning communities: Twenty-one designs to building an online collaborative learning community. Westport: CT: Libraries Unlimited.

Yazici, H.J. (2004). Student Perceptions of Collaborative Learning in Operations Management Classes . Journal of Education for Business, 80(2): 110-118.

\section{María Pilar Flores-Asenjo}

Licenciada en Ciencias Económicas y Empresariales en la Facultad de Económicas de la Universidad de Valencia. Doctora en Economía y Ciencias Sociales por la Universidad Politécnica de Valencia en 2008. Profesora asociada de la Facultad de Economía de la Universidad de Valencia (1998-2001). Profesora del Departamento de Administración y Dirección de Empresas de la Universidad Católica San Antonio de Murcia desde 2001 hasta la fecha. Miembro del Grupo de Investigación sobre líneas de comportamientos de compra disfuncionales y satisfacción e insatisfacción del consumidor, Marketing turístico y turismo sostenible. 